\title{
Taste-aroma interactions in a ternary system: A model of fruitiness perception in sucrose/acid solutions
}

\author{
JOHANN C. PFEIFFER, JOANNE HORT, TRACEY A. HOLLOWOOD, and ANDREW J. TAYLOR \\ University of Nottingham, Sutton Bonington, Loughborough, England
}

\begin{abstract}
Cross-modal interactions between aroma, sweetness, and acidity were studied. A series of samples was presented to trained panelists who assessed strawberry flavor intensity using magnitude estimation with a reference modulus. The delivery of aroma stimuli from the different solutions was measured by monitoring exhaled breath using atmospheric pressure chemical ionization-mass spectrometry to determine whether there were any physicochemical effects on volatile release; no significant differences were noted. Three-dimensional predictive models were built to describe perceived strawberry flavor intensity as a function of concentrations of sucrose, acid, and volatiles. Analysis of the data identified two groups of panelists with different responses: For Group 1, increasing sucrose and/or acid levels also increased the perceived flavor intensity. For Group 2, changing sucrose concentrations had little effect, but increasing acid and/or volatile levels did. The results show different effects of organic and inorganic acids on perception, as well as clear interactions between the modalities of taste (sugar and acid) and aroma. The clustering of panelists' responses suggests that this phenomenon may depend on prior associations between the fruity flavor and the tastants.
\end{abstract}

Although cross-modal effects have been studied for many of the senses, such as vision, hearing, and touch (Calvert, Spence, \& Stein, 2004), the discovery of cross-modal effects in flavor perception is relatively new. There is much anecdotal evidence of cross-modal effects with food, since small amounts of tastants such as sugar, salt, or monosodium glutamate are known to enhance the overall flavor of foods. However, these compounds can also interact with the food aroma compounds in a physicochemical way, and it has been difficult to determine whether the basis of interaction was physicochemical or cross-modal. It is now established that significant physicochemical changes between tastants and aroma stimuli only occur at solute concentrations above $100 \mathrm{~g} / \mathrm{L}$ (Friel, Linforth, \& Taylor, 2000), and the advent of methods for measuring both volatile and tastant delivery in vivo allows further checks to be made to rule out any physicochemical effects.

In our laboratory, we have studied taste-aroma-viscosity interactions (Cook, Davidson, Linforth, \& Taylor, 2004; Cook, Hollowood, Linforth, \& Taylor, 2002, 2003; Cook, Hollowood, Pettelot, \& Taylor, 2003; Cook, Linforth, \& Taylor, 2003; Davidson, Linforth, Hollowood, \& Taylor, 1999; Hollowood, Davidson, DeGroot, Linforth, \& Taylor, 2002; Hollowood, Linforth, \& Taylor, 2002; Hort \& Hollowood, 2004) and have used in vivo analyses to en-

The authors thank Masterfoods, Slough, U.K., for their financial support. Correspondence concerning this article should be addressed to J. Hort, Division of Food Sciences, University of Nottingham, Sutton Bonington Campus, Loughborough LE12 5RD, England (e-mail: joanne.hort@nottingham.ac.uk). sure that the stimuli delivered to the receptors are not significantly affected by components present in the sample (e.g., hydrocolloids, sucrose, or salt). Potentially, these solutes affect the partition behavior and thus the release (and perception) of a volatile compound from a solution, and it is therefore essential to measure this effect to establish whether it is significant. Dalton, Doolittle, Nagata, and Breslin (2000) used a benzaldehyde/saccharin system to study cross-modal taste-aroma interactions while avoiding physicochemical interactions. Since saccharin has a high sweetness intensity, the amounts used would have had no effect on the partition behavior of benzaldehyde (almond flavor). To test for a cross-modal effect, benzaldehyde was delivered orthonasally at a subthreshold concentration while either water or a subthreshold saccharin solution was presented orally. Panelists were asked whether they could or could not detect any benzaldehyde aroma, making the sensory evaluation simple, and therefore robust. The aroma was detectable for a significant number of participants when sensed in combination with a subthreshold tastant - in other words, the presence of saccharin at a subthreshold level led to a decrease in the detection threshold for benzaldehyde, demonstrating a cross-modal effect.

This phenomenon has also been reported at a suprathreshold level, especially with sugars and acids. For instance, Cayeux and Mercier (2003) revealed an enhanced perceived intensity of apple and lemon flavor in the presence of a sour taste, although no changes took place in in-nose volatile release, which, according to the authors, ruled out any physicochemical interactions. Other reports have lent weight to the idea that cross-modal interactions 
occur, but they have not eliminated the potential physicochemical factor (e.g., Bonnans \& Noble, 1993; Kuo, Pangborn, \& Noble, 1993; Valdés, Simone, \& Hinreiner, 1956). Generally speaking, cross-modal effects have been noted with congruent tastants and volatiles (i.e., with familiar flavor pairings, such as salt and savory flavors; Cook, Linforth, \& Taylor, 2003). Evidence from brain imaging studies has also indicated that signals from the taste and aroma modalities are integrated in the orbitofrontal cortex (Rolls \& Baylis, 1994; Small et al., 2004).

Practically all of the studies listed above have used binary flavor systems, and the objective of the present research was to study potential cross-modal effects in a ternary system typical of a fruit beverage (i.e., containing sweetness, acidity, and a strawberry aroma mixture). To avoid any confusion, we define the aroma stimulus as the mixture of volatile compounds present in the sample, and the term flavor indicates the overall combination of olfactive, gustative, and mouth-feel sensations. Strawberry flavor (or strawberryness) only refers to the olfactory perceptual dimension when evaluating the samples. One type of sweetener (sucrose) and one type of aroma stimulus (strawberry) was investigated, whereas for acidity, the most commonly used beverage acids - citric, malic, lactic, and phosphoric acids - were selected. Initially, experiments focused on whether taste-aroma interactions could be detected in the ternary system and whether any confounding physicochemical effects were present, by measuring the in-nose volatile release via atmospheric pressure chemical ionization-mass spectrometry (APcI-MS). Subsequently, further experimentation was carried out, and the data were used to develop models that could predict perceived flavor intensity in relation to the amounts of tastants and aroma present.

\section{EXPERIMENT 1}

\section{Method}

\section{Participants}

Panelists ( 9 females/2 males, between 34 and 64 years of age) from the University of Nottingham external sensory panel were chosen, on the basis of availability and previous training in magnitude estimation, to evaluate strawberry flavor intensity. Three students of the University of Nottingham volunteered for the instrumental analysis. All panelists were naive as to the identity of the substances used and the purpose of the experiments.

\footnotetext{
Materials

Samples consisted of combinations of sucrose (Fisher Scientific, Loughborough, U.K.), acid, and a commercial strawberry aroma stimulus (Firmenich SA, Geneva, Switzerland; Strawberry 502301T). Acids selected for investigation were citric acid (Lancaster Synthesis, Lancaster, U.K.; $\geq 99 \%$ pure), lactic acid (Aldrich Chemicals, Poole, U.K.; $85 \%$ w/w syrup, $98 \%$ pure), DL malic acid (Aldrich Chemicals; 99\% pure), and phosphoric acid (Aldrich Chemicals; concentration in water $85 \%, \geq 99 \%$ pure). Colorless samples were made using still spring water (Brecon Beacons Natural Waters, U.K.) as solvent. Stock solutions were prepared $4 \mathrm{~h}$ prior to testing and roller mixed for $2 \mathrm{~h}$ to ensure complete dissolution and homogeneity. Sample compositions are represented in Table 1.
}

Table 1 Composition of Samples Investigated in Experiment 1

\begin{tabular}{cccc}
\hline Sample Number & Sucrose $(\% \mathrm{w} / \mathrm{w})$ & Acid $(\% \mathrm{w} / \mathrm{v})^{*}$ & Aroma $(\% \mathrm{v} / \mathrm{v})$ \\
\hline 1 & 0 & 0 & 0.18 \\
2 & 10 & 0 & 0.18 \\
3 & 0 & 0.3 & 0.18 \\
4 & 3.4 & 0.125 & 0.18 \\
5 & 10 & 0.3 & 0.18 \\
\hline
\end{tabular}

${ }^{*}$ Four acids were used (citric, malic, lactic, and phosphoric), resulting in a total of 14 samples investigated (Sample $1+$ Sample $2+$ [Samples 3, $4,5] * 4)$.

\section{Sensory Procedure}

Strawberry flavor intensity was quantified using a magnitude estimation technique with a reference modulus (ISO 11056), based on data collected with the computerized data acquisition system FIZZ (Biosystèmes, Couternon, France). The reference modulus, containing $0.18 \% \mathrm{v} / \mathrm{v}$ aroma stimulus, $3.4 \% \mathrm{w} / \mathrm{w}$ sucrose, and $0.125 \% \mathrm{w} / \mathrm{v}$ citric acid, was chosen for its moderate and typical strawberry flavor intensity. During an initial training session, the panelists became familiar with the strawberry flavor, the sample range, and the reference modulus, which was assigned a score of 100 in terms of strawberry flavor intensity.

In two subsequent sessions, the panelists were asked to evaluate and score the strawberry flavor intensity of the various samples relative to the reference, which was presented with each sample. The instructions emphasized that only strawberry flavor intensity was to be judged and that hedonic or any other attributes were to be disregarded. To assess panelist consistency, it was decided to include a blind coded reference modulus in each session, resulting in a total of 14 samples (Table 1) and 2 blind reference moduli to evaluate across the two sessions. Eight samples were assessed in duplicate in each session. The panelists evaluated samples in sets of four with $45-\mathrm{sec}$ breaks between samples and 15-min breaks between sets. Presentation orders were counterbalanced (within each session) using a Williams square (Edwards, 1968). All samples were swallowed to simulate typical consumption of foods and beverages (Buettner, Beer, Hannig, \& Settles, 2001; Burdach, Kroeze, \& Köster, 1984; Murphy \& Cain, 1980), and a palate cleanser of water and crackers was provided between samples. All stimuli were presented in aliquots of $20 \mathrm{~mL}$, contained in $25-\mathrm{mL}$ transparent disposable plastic cups (with a three-digit code), which were filled $1 \mathrm{~h}$ before the start of the experiment to allow temperature equilibrium among the samples. All testing was performed at room temperature $\left(\sim 20^{\circ} \mathrm{C}\right)$ in an air-conditioned room under Northern Hemisphere daylight and in individual booths.

\section{Volatile Release Measurements}

APcI-MS was used to monitor the volatile release in nose during drinking. The method is well described in the literature (Taylor \& Linforth, 2003; Taylor, Linforth, Harvey, \& Blake, 2000). The main compounds present in the strawberry aroma stimulus were identified as esters. Because monitoring too many ions decreases the sensitivity of the machine, it was decided to follow only the release of the volatiles most characteristic of the strawberry aroma: ethyl acetate, ethyl butyrate, and ethyl caproate, with ion $\mathrm{m} / \mathrm{z}$ values of 89,117 , and 145 , respectively. Three panelists were asked to consume four replicates of each sample over four separate sessions, one for each acid. To assess the effect of acid type on volatile release, Sample 4 with citric acid was included within the malic, lactic, and phosphoric acid sessions.

\section{Data Analysis}

One-way ANOVA on the reference modulus scores was performed to check for panel consistency. Two-way ANOVA and, where ap- 
propriate, Student/Newman-Keuls multiple-comparison tests $(\alpha=$ $.05)$ were applied to the replicate data in order to test for significant differences in perceived strawberry flavor intensity between samples and to account for panel dissimilarities. Data from the APcI-MS experiments were analyzed via ANOVA on individual panelists.

\section{Results}

\section{Influence of Sucrose and Acid on the Perception of Strawberry Flavor Intensity}

Statistical analysis of the reference modulus revealed inconsistent scoring from 2 panelists (1 male of 64 and 1 female of 58 years of age); their data were excluded from further analysis. Two-way ANOVA (panelists $\times$ samples) indicated that significant differences existed between the strawberry flavor intensity of the samples for each acid as well as within the panel. The latter variation is probably due to variations in the use of the scale.

The results of the multiple-comparison tests are summarized in Table 2. For citric, malic, and phosphoric acids, there were significant differences between all of the samples, with the exception of Samples 2 and 3 (representing high sucrose/no acid and no sucrose/high acid, respectively). Samples containing lactic acid were more difficult to differentiate, since only three subsets were obtained; however, the extreme samples (no sucrose/no acid and high sucrose/high acid) differed significantly.

The mean sensory scores (Figure 1) showed a similar pattern for the four acids: The more sucrose and/or acid present in the formulation, the more intense the strawberry flavor intensity was rated. This was further confirmed by the statistical analysis of the whole data set and the groupings identified by the Student/Newman-Keuls tests, which are also shown on Figure 1. The most noticeable difference in intensity was between Samples 1 and 5, which contained no sucrose/no acid and high sucrose/ high acid, respectively. In addition, the analysis of scores highlighted synergistic effects of sucrose and acid. The sum of intensity ratings from Sample 2 (10\% sucrose) and Sample $3(0.3 \%$ acid $)$ was inferior to the Sample 5 score, which contained both sugar and acid (10\% sucrose $/ 0.3 \%$ acid), implying that strawberryness was perceived more intensely when sucrose and acid were presented together than when each tastant was presented alone.

\section{Impact of Sucrose/Acid Levels and Acid Type on Aroma Release}

For convenience, Figure 2 represents data from 1 panelist only, since results were similar for the other 2 panelists, although there was some variation in the concentrations of volatiles found in nose, probably due to personal physiology and behavior patterns during drinking (Buettner et al., 2001; Buettner \& Schieberle, 2000a, 2000b; Hodgson, Linforth, \& Taylor, 2003). For sucrose and each acid independently, there was no significant difference between samples with regard to the concentration of volatiles released for the three esters monitored during drinking. Neither was any significant difference observed between citric acid and the three other acids for Sample 4, suggesting that the differences in acid concentrations seen in Figure 2 are likely to be related to physiological changes of the panelists across the four sessions or to the different operating conditions of the APcI-MS, since minor fluctuations in signal intensity do occur over a period of several weeks.

\section{Discussion}

Experiment 1 indicated the presence of taste-aroma interactions within the limits of the system investigated. Although the volatile concentration remained constant in all samples, the perceived strawberry flavor intensity increased with an increase in acid or sucrose content. This effect was even stronger when the two tastants were presented in combination, suggesting super additivity. Since the release of the three volatiles monitored was unaffected by the presence of high amounts of sucrose and/or acid, the results obtained rule out physicochemical interactions, suggesting that perceptual, cross-modal interactions are responsible. In this experiment, only a few discrete samples were investigated. The purpose of Experiment 2 was to determine whether analogous results would be obtained throughout the whole sample space - that is, if the effects of sucrose and acid on perceived flavor intensity follow a logical pattern. Experiment 2 especially aimed to model the perceptual feedback in terms of sample composition.

\section{EXPERIMENT 2}

\section{Method}

\section{Participants and Materials}

The 2 inconsistent panelists from Experiment 1 were excluded, so 2 additional female panelists were recruited for Experiment 2, making 11 panelists overall. The materials were identical to those used in Experiment 1.

\section{Experimental Design}

Response surface methodology (RSM) was chosen as the tool to investigate and quantify the relationship between the controlled

Table 2

The Effects of Sucrose and Acid on Perceived Strawberry Flavor Intensity: Subsets of Significantly Different Samples for Each Acid Separately

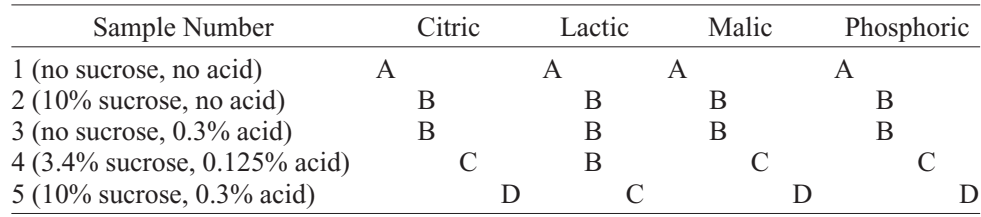

Note-Sucrose is measured in $\% \mathrm{w} / \mathrm{w}$, and acid in $\% \mathrm{w} / \mathrm{v}$. 


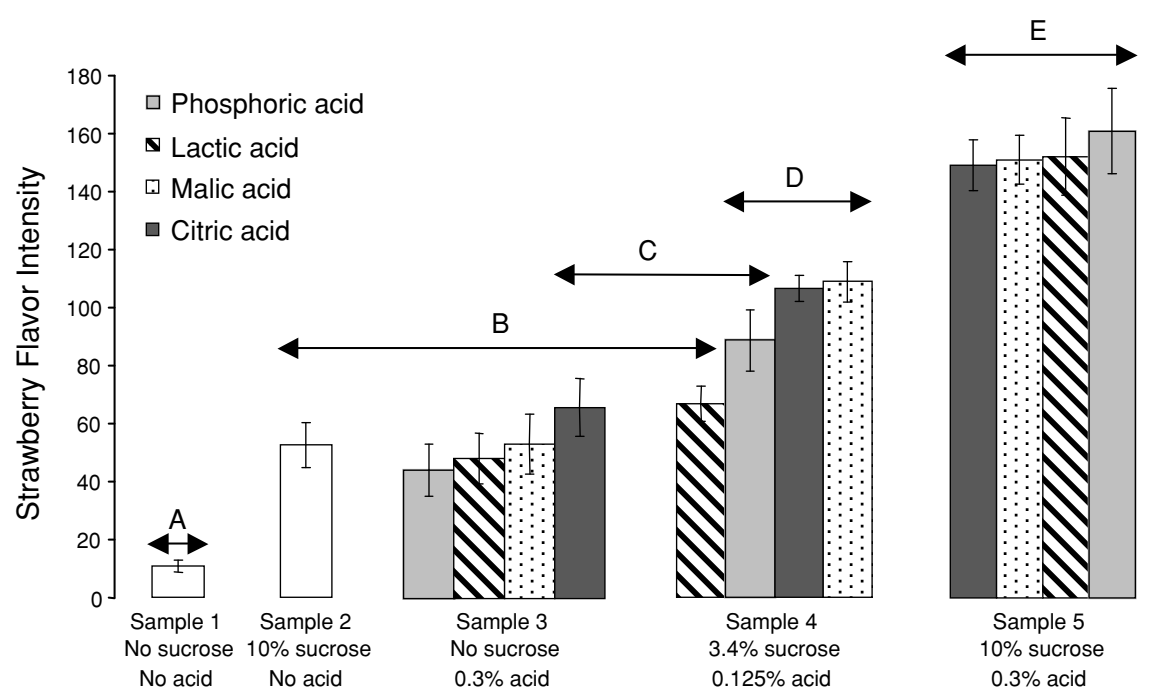

Figure 1. Perceived strawberry flavor intensity of the various stimuli from Table 1 for citric, lactic, malic, and phosphoric acids. Data are the grand means of 9 panelists/2 sensory replicates \pm standard error. The different letters indicate subsets of significantly different samples obtained from Student/Newman-Keuls multiple-comparison tests. Sucrose was measured as $\% \mathrm{w} / \mathrm{w}$ and acid as $\% \mathrm{w} / \mathrm{v}$.

parameters (sucrose, acid, and aroma stimulus concentrations) and the response measured (strawberry flavor intensity). RSM is a set of techniques designed to characterize this relationship by producing mathematical models that identify which factors are significant in terms of the response. Design-Expert software, Version 6.0 (StatEase Corp., Minneapolis, MN), was used to generate a D-optimal design. Using a specific algorithm that minimizes the variance associated with the estimates of specified model coefficients, the
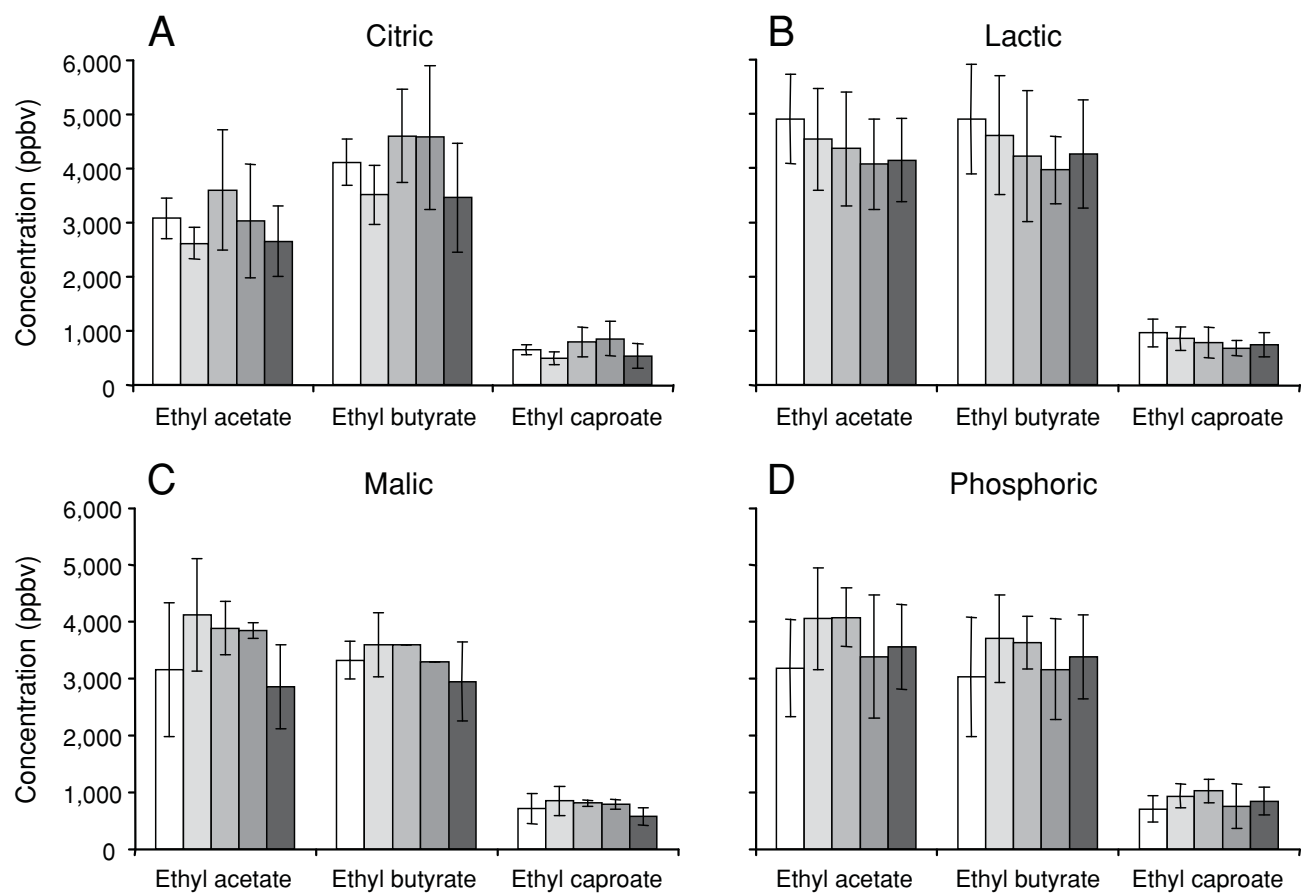

$\square$ Sample $1 \quad \square$ Sample 2 a Sample 3 a Sample $4 \quad \square$ Sample 5

Figure 2. First exhalation concentrations of the monitored esters during consumption of stimuli from Table 1 (A, citric acid; B, lactic acid; C, malic acid; D, phosphoric acid). Data are the means of four replicates \pm standard deviations from 1 panelist. 


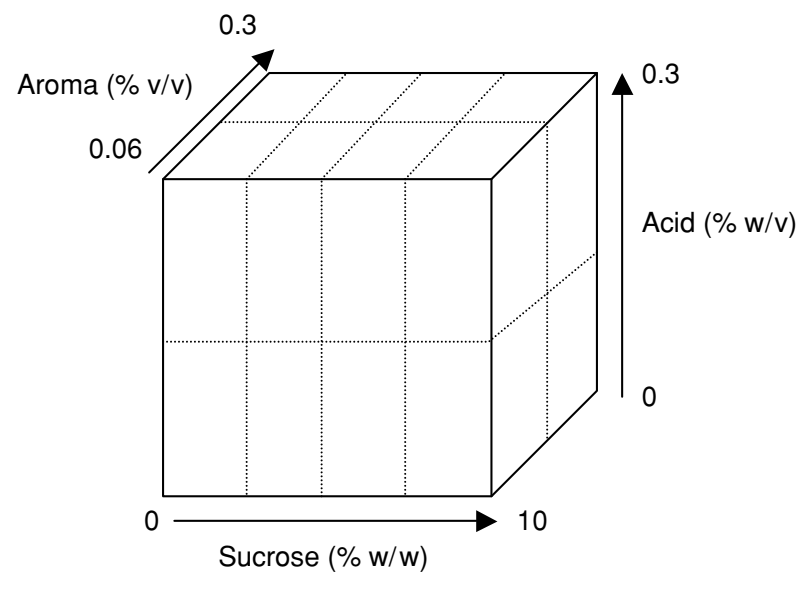

Figure 3. Graphical representation of the sample space to model the influence of sucrose, acid, and aroma stimulus concentrations on the perception of strawberry flavor intensity.

D-optimal design chooses an ideal subset of all possible combinations. Thus, as opposed to a three-factorial response surface design, this design makes an appropriate choice for sensory testing by considerably reducing the number of design points - that is, the number of samples to evaluate.

The limits of the system investigated were $0-10 \%(\mathrm{w} / \mathrm{w})$ sucrose, $0-0.3 \%(\mathrm{w} / \mathrm{v})$ acid, and $0.06-0.3 \%(\mathrm{v} / \mathrm{v})$ strawberry aroma. The total sample space is represented in Figure 3. The D-optimal design suggested collecting data from 18 combinations of acid, sucrose, and aroma stimulus for citric and malic acids and 19 combinations for lactic and phosphoric acids (because of the design), plus a further four experimental replicates in each case. An additional set of 15 samples, based on time availability during sessions and spread across the original sample space, was evaluated separately to provide a validation data set for each acid.

\section{Sensory Procedure}

Samples suggested by the D-optimal design that contained no acid were only assessed once by the panelists, and not separately for each acid set, which gave a total of 136 samples to be assessed. The samples (test set and following validation set) were randomly allocated to 17 two-hour sensory data collection sessions. Eight samples were assessed in duplicate in each of the sessions. An additional blind coded reference modulus was included in seven of the sessions to assess the consistency of the panelists. Samples were assessed under the same conditions and using the same protocols outlined previously for Experiment 1 .

\section{Data Analysis}

Two different strategies of data analysis were selected: first, examining a global response (i.e., the grand mean of all panelists), and second, taking into consideration each panelist's individual means of the sensory replicates. In each case, the Design-Expert program was used to generate models to explain variations of perceived strawberry flavor intensity as a function of sucrose, acid, and aroma concentrations. The inclusion of significant model terms was determined through ANOVA and additional diagnostic tests to identify the mathematical model that best explained the data. In each case, consideration of the best-fit equations (those with the highest $R^{2} \mathrm{~s}$ ) and those with the best predictive abilities (lowest predicted residual sum of squares and highest predictive $R^{2}$ ) aided the selection of the final models.

\section{Results}

\section{Influence of Sucrose, Acid, and Aroma Levels on the Perception of Strawberry Flavor Intensity}

Using the global-mean approach, highly significant ( $p<$ .0001) low-order polynomial (quadratic) models were successfully generated in Design-Expert that showed a nonlinear relationship between strawberry flavor intensity perception and sucrose, acid, and aroma stimulus concentrations. Table 3 summarizes for each acid the model produced and its associated statistics: adjusted $R^{2}$, predicted $R^{2}$, and $a d-$ equate precision, a measure of signal-to-noise ratio (values higher than 4 indicate adequate precision, as suggested for Design-Expert 6.0). There was no significant lack of fit (variation of the data around the fitted model). These parameters indicated the robustness of the constructed models, which was further confirmed when experimental values were plotted against predicted values from the models (Figure 4). The models also closely predicted the actual strawberry flavor intensity values obtained from the panel for the validation data sets (Figure 4), adding further evidence of the robustness of the models.

The lack of interaction terms between aroma and sucrose and between aroma and acid implies that the aroma stimulus concentration had no significant influence on the shape of the models, which is defined for a given model by sets of lines of equal perceived intensity, commonly referred to as contours. This further indicates that the position of these contours remains the same for any aroma concentration within the limits of the system. However, as expected, the aroma stimulus level did affect the perceived strawberry flavor intensity, since aroma is a significant term. The interaction term between sucrose and acid for the four acids investigated reveals the important connection of these two factors: The relationship between perceived strawberry flavor intensity and sucrose concentration is dependent on acid level, and conversely the re-

Table 3

Predicted Perceived Strawberry Flavor Intensity as a Function of Sample Composition and Acid Type for a Global-Mean Strategy

\begin{tabular}{|c|c|c|c|c|c|c|c|c|c|c|c|}
\hline \multirow[b]{2}{*}{ Acid } & \multicolumn{8}{|c|}{ Perceived Strawberry Flavor Intensity = } & \multirow[b]{2}{*}{ Adjusted $R^{2}$} & \multirow[b]{2}{*}{ Predicted $R^{2}$} & \multirow[b]{2}{*}{$\begin{array}{l}\text { Adequate } \\
\text { Precision }\end{array}$} \\
\hline & Constant & * Sucrose & * Acid & * Aroma & * Sucrose ${ }^{2}$ & $* \operatorname{Acid}^{2}$ & $\begin{array}{c}* \text { Sucrose } \\
* \text { Acid }\end{array}$ & * Aroma $^{2}$ & & & \\
\hline$\overline{\text { Citric }}$ & -15.4 & 11.0 & 439 & 154 & -0.611 & -871 & 14.7 & & .97 & .96 & 35.9 \\
\hline Lactic & -10.8 & 11.4 & 268 & 139 & -0.688 & -436 & 17.7 & & .96 & .94 & 28.7 \\
\hline Malic & -10.1 & 10.8 & 463 & 140 & -0.631 & $-1,026$ & 14.8 & & .97 & .95 & 31.7 \\
\hline Phosphoric & -30.4 & 5.6 & 520 & 447 & & $-1,523$ & 19.2 & -866 & .96 & .93 & 25.6 \\
\hline
\end{tabular}

Note-Each row represents a polynomial equation, with values representing the contribution of each factor. The specific units for the numerical estimates are sucrose, $\% \mathrm{w} / \mathrm{w}$; acid, $\% \mathrm{w} / \mathrm{v}$; and aroma stimulus, $\% \mathrm{v} / \mathrm{v}$. 

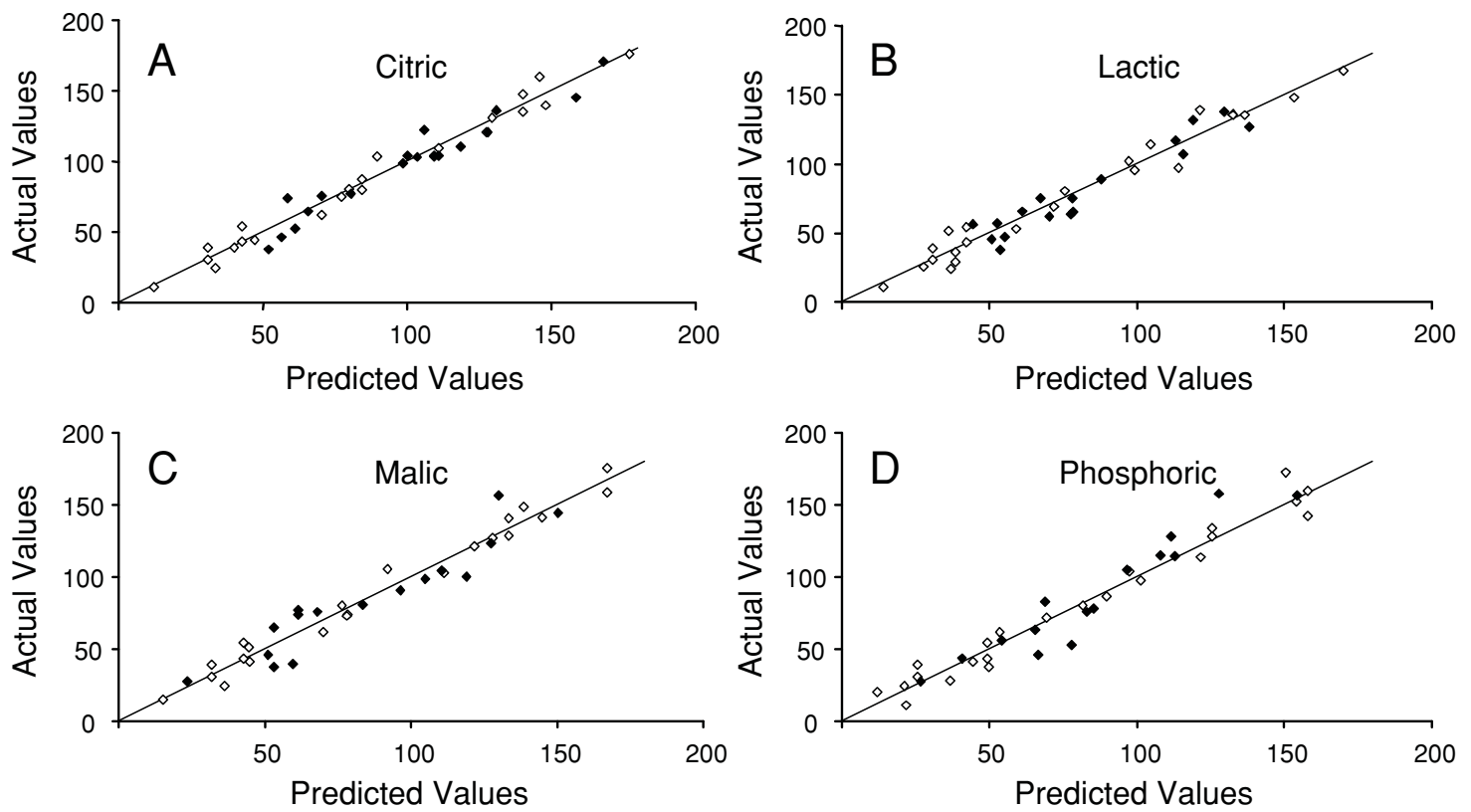

- Model point $\cdot$ Validation points

Figure 4. Perceived strawberry flavor intensity: A comparison of predicted values from the models ( $x$-axis) and experimental results ( $y$-axis). Both points used to build the models (open diamonds) and points used as the validation set (filled diamonds) are represented.

lationship between perceived strawberry flavor intensity and acid concentration is also dependent on sucrose level. Figure 5 illustrates this statement with slices of the threedimensional models in which each contour represents a constant perceived intensity value $(60,80,100, \ldots)$ obtained with different formulations of sucrose and acid concentrations.

\section{Influence of Acid Type on the Model Response}

Analysis of the contour plots obtained from the models showed three different profiles. With citric and malic acids, the models are almost symmetrical, suggesting that both sucrose and acid levels equally influence the strawberry flavor intensity: Increasing levels progressively increases the perceived intensity. On the other hand, when using lactic acid, this relative balance was shifted: Flavor perception was less affected by the acid level than by the sucrose level; that is, for a given increase in sucrose level, the acid needed to be increased twofold to provide the same increase in perception. The third behavior concerned the model with phosphoric acid, for which the ellipsoids are almost horizontal; for each sucrose level, increasing the acid content increased the flavor perception until a limit concentration was reached, and thereafter the perceived strawberry flavor intensity decreased with an increase in acid.

\section{Influence of the Panelists}

As has been previously shown, the models using the global-mean strategy were robust. However, to investigate any effect of panelists (i.e., to take into account the variation between panelists), models were generated using the data from individual panelists. Although the models were still highly significant $(p<.0001)$, they also included panelist as a significant term $(p<.01)$. Closer observation of the data and interaction plots indicated a difference in response behavior between the panelists. The panel appeared to be split into two groups.

Data analysis of Group 1. This group included 8 panelists. In new models $(p<.0001)$ generated using only data from this group, the panelist term was no longer significant and was, therefore, not included in the final equations. Associated model statistics are summarized in Table 4, and slices throughout the three-dimensional models at $0.18 \%$ aroma stimulus concentration are shown in Figure 6. Although involving different significant terms, these models are very similar to those obtained in the previous section (Figure 5), and the same comments on their shapes can be made: Both acid and sucrose influence strawberry flavor perception, and the effects are acid-type dependent. Predicted values plotted against experimental values (Figure 7 ) gave predicted $R^{2} \mathrm{~s}$ of $.75, .70, .73$, and .70 , respectively, for citric, lactic, malic, and phosphoric acids; although these values are not as high as those in the previous section, they are still satisfactory. The validating data set was composed of sample means for the 8 panelists, since there was no significant difference in scoring between panelists. Every single value was very close to its predicted value (Figure 7), which implies that 

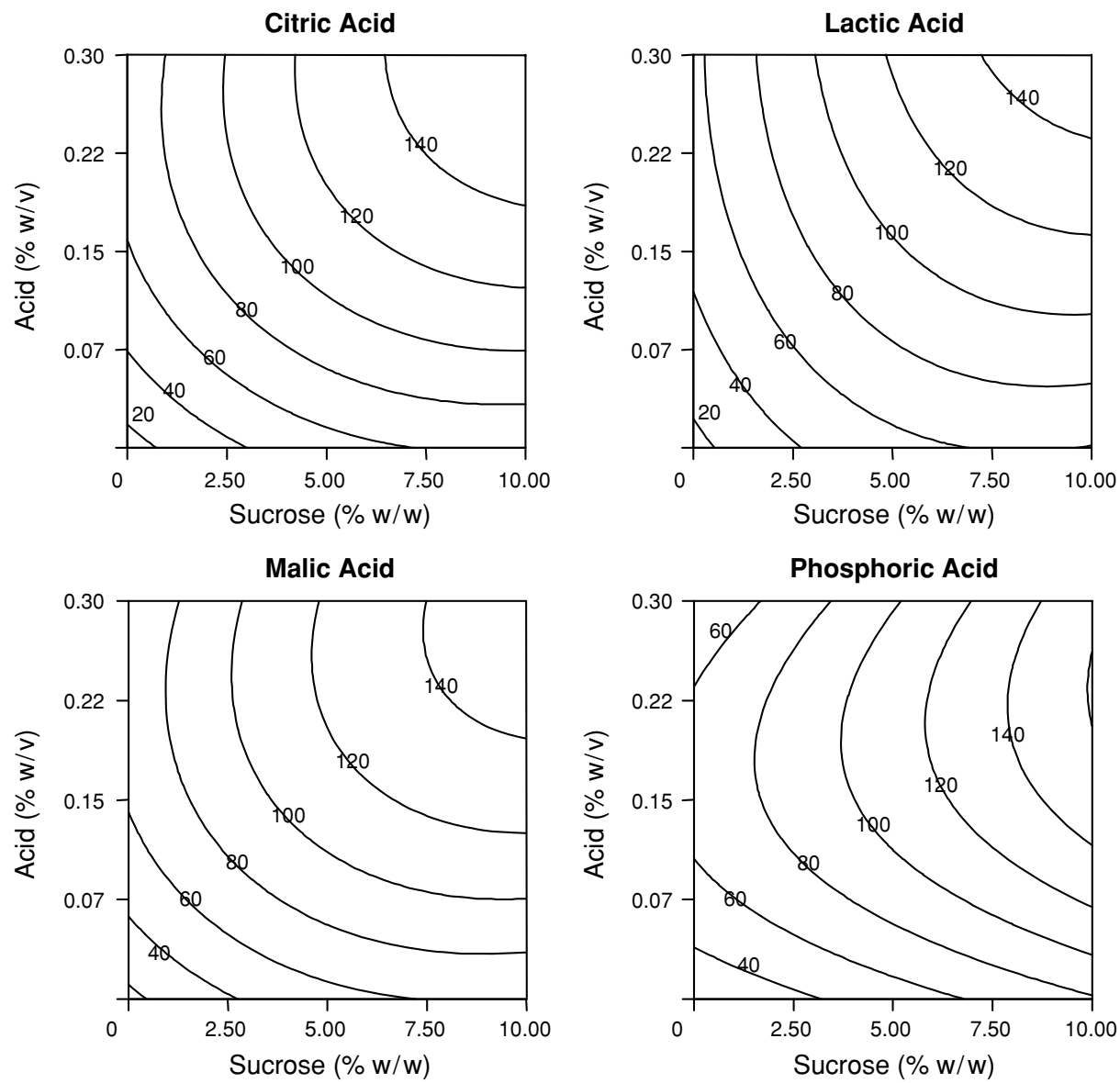

Figure 5. Two-dimensional contour plots derived from the generated models. Contours represent perceived strawberry flavor intensity as a function of the experimental factors - that is, the different types of acids and the various amounts of acid $(\% \mathrm{w} / \mathrm{v})$ and sucrose $(\% \mathrm{w} / \mathrm{w})$ for a fixed aroma stimulus concentration $(0.18 \% \mathrm{v} / \mathrm{v})$.

the designed models explained the variation in the sensory results satisfactorily.

Data analysis of Group 2. This group included 3 panelists. The models were highly significant $(p<.0001)$ with no lack of fit, but unlike with the models for Group 1, panelist was still a significant factor, because of individual differences in intensity scoring rather than differences in behavior among the panelists. This difference is highlighted in Tables 5 and 6, where the constant values and acid contributions differ from panelist to panelist, but other terms for significant factors remain unchanged. The results also indicate dissimilarities between the four models for this group. Indeed, the models describing citric, lactic, and malic acids do not involve sucrose as a significant term, in contrast with the phosphoric acid model (Table 6); however, even in the latter model, the sucrose contribution is very small in comparison with the acid effect. This result is of considerable importance, because it

Table 4

Predicted Perceived Strawberry Flavor Intensity as a Function of Sample Composition and Acid Type for Group 1

\begin{tabular}{|c|c|c|c|c|c|c|c|c|c|c|c|c|}
\hline \multirow[b]{2}{*}{ Acid } & \multicolumn{9}{|c|}{ Square Root (Perceived Strawberry Flavor Intensity $+k)=$} & \multirow[b]{2}{*}{ Adjusted $R^{2}$} & \multirow[b]{2}{*}{ Predicted $R^{2}$} & \multirow[b]{2}{*}{$\begin{array}{l}\text { Adequate } \\
\text { Precision }\end{array}$} \\
\hline & $k$ & Constant & * Sucrose & * Acid & * Aroma & * Sucrose ${ }^{2}$ & * $\operatorname{Acid}^{2}$ & $\begin{array}{c}* \text { Sucrose } \\
* \text { Acid }\end{array}$ & $\begin{array}{c}* \text { Acid } \\
* \text { Aroma }\end{array}$ & & & \\
\hline Citric & 2.25 & 1.45 & 1.02 & 38.7 & 6.13 & -0.050 & -67.2 & & & .76 & .75 & 31.3 \\
\hline Lactic & 2.50 & 1.0 & & & 7.9 & & & & & .71 & .70 & 32.7 \\
\hline Malic & 3.00 & 2.14 & 0.99 & 38.5 & 5.08 & -0.056 & -84.7 & 0.58 & & .75 & .73 & 26.5 \\
\hline Phosphoric & 2.75 & 1.20 & 0.83 & 42.6 & 8.86 & -0.036 & -105 & 1.03 & -27.9 & .72 & .70 & 21.6 \\
\hline
\end{tabular}

Note-Each row represents a polynomial equation, with values representing the contribution of each factor. The specific units for numerical estimates are sucrose, $\% \mathrm{w} / \mathrm{w}$; acid, $\% \mathrm{w} / \mathrm{v}$; and aroma stimulus, $\% \mathrm{v} / \mathrm{v}$. 
Citric Acid

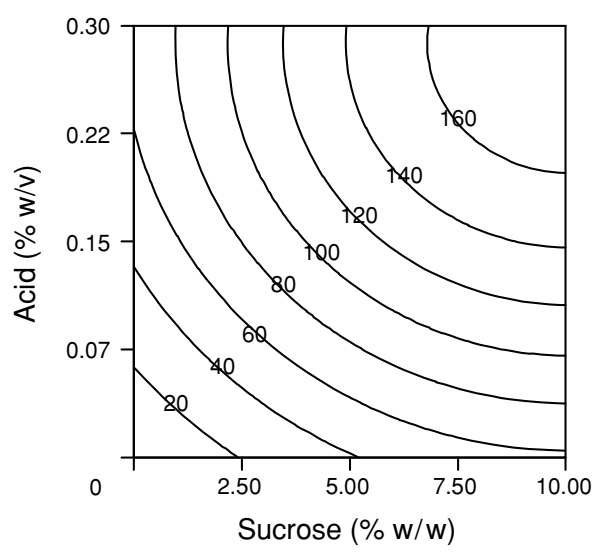

Malic Acid

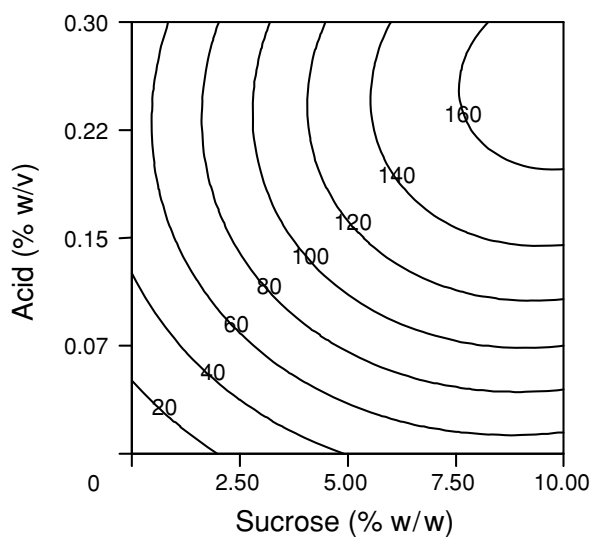

Lactic Acid

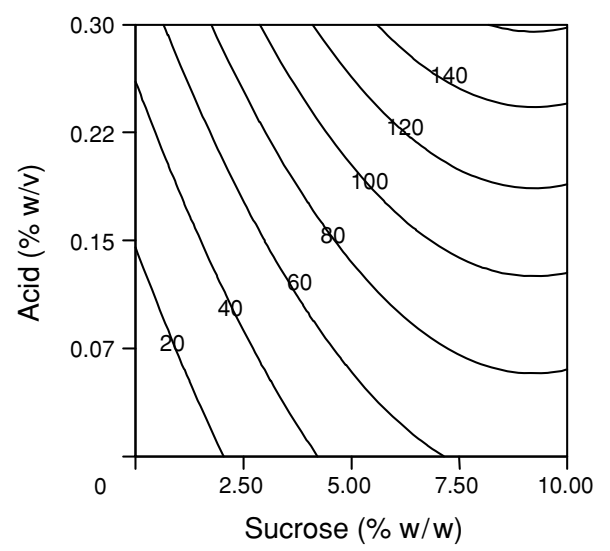

Phosphoric Acid

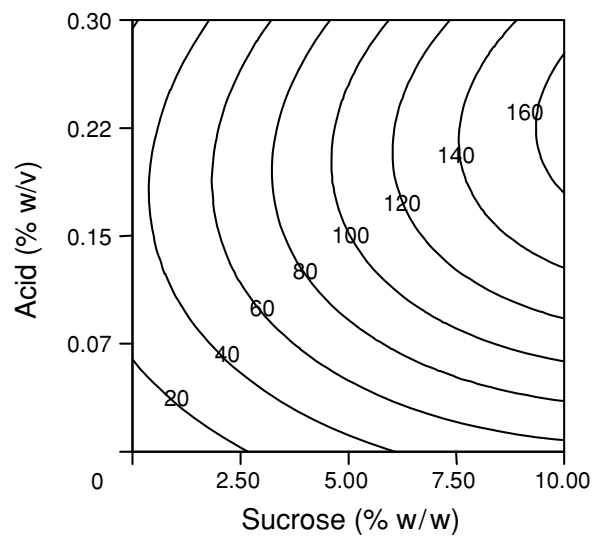

Figure 6. Two-dimensional contour plots derived from the models obtained with Group 1. Contours represent perceived strawberry flavor intensity as a function of the experimental factors- that is, the different types of acids and various amounts of acid $(\% \mathrm{w} / \mathrm{v})$ and sucrose $(\% \mathrm{w} / \mathrm{w})$ for a fixed aroma stimulus concentration $(0.18 \% \mathrm{v} / \mathrm{v})$.

suggests that for these 3 panelists, perceived strawberry flavor is scarcely driven by the amount of sucrose in the formulation, but mainly by the presence of acid and the aroma stimulus. For citric, lactic, and malic acids, therefore, the models (not shown) change from three-dimensional to two-dimensional, with only aroma and acid factors affecting flavor perception. Again, the models for citric and malic acids are similar with respect to shape and intensity, and they produce a similar trend, the presence of an optimum acid concentration (around $0.2 \% \mathrm{w} / \mathrm{v}$ ) at which the influence on strawberry flavor intensity is at a maximum. For lactic acid, increasing either acid level or aroma has a similar effect on increasing perceived intensity. Although they vary in intensity scores, the models for the 3 panelists with phosphoric acid reflect the same behavior that was observed with Group 1: The maximum perceived flavor intensity was reached at an acid concentration below the maximum level.

\section{Discussion}

The results indicate that, over the ranges tested in these experiments and for the majority of the panelists, sucrose and acids contributed more than just sweetness and sourness: They played a critical role in the perception of strawberry flavor intensity.

Both citric and malic acids showed an enhancement effect similar to that of sucrose. However, the use of lactic and phosphoric acids led to different results, thereby introducing the idea that the combined impact of acid and sucrose is acid-type dependent. With phosphoric acid, there is an optimum concentration $\left(C_{\text {limit }}\right)$ after which the perceived intensity starts to decrease. One hypothesis is that up to $C_{\text {limit }}$, phosphoric acid contributed to the enhancement in a manner similar to citric or malic acid. It is important, however, to notice that the panelists verbally reported greater sourness and astringency for the maximum concentration of phosphoric acid than for the other acids. Thus, perhaps above $C_{\text {limit }}$ the different in-mouth sensations exhibited by phosphoric acid indirectly resulted in a decrease in perceived intensity by masking the strawberry flavor, inducing the reverse effect observed. Unique taste-taste interactions between sucrose and phosphoric acid or a different taste transduction mechanism specific to strong acids (DeSim- 

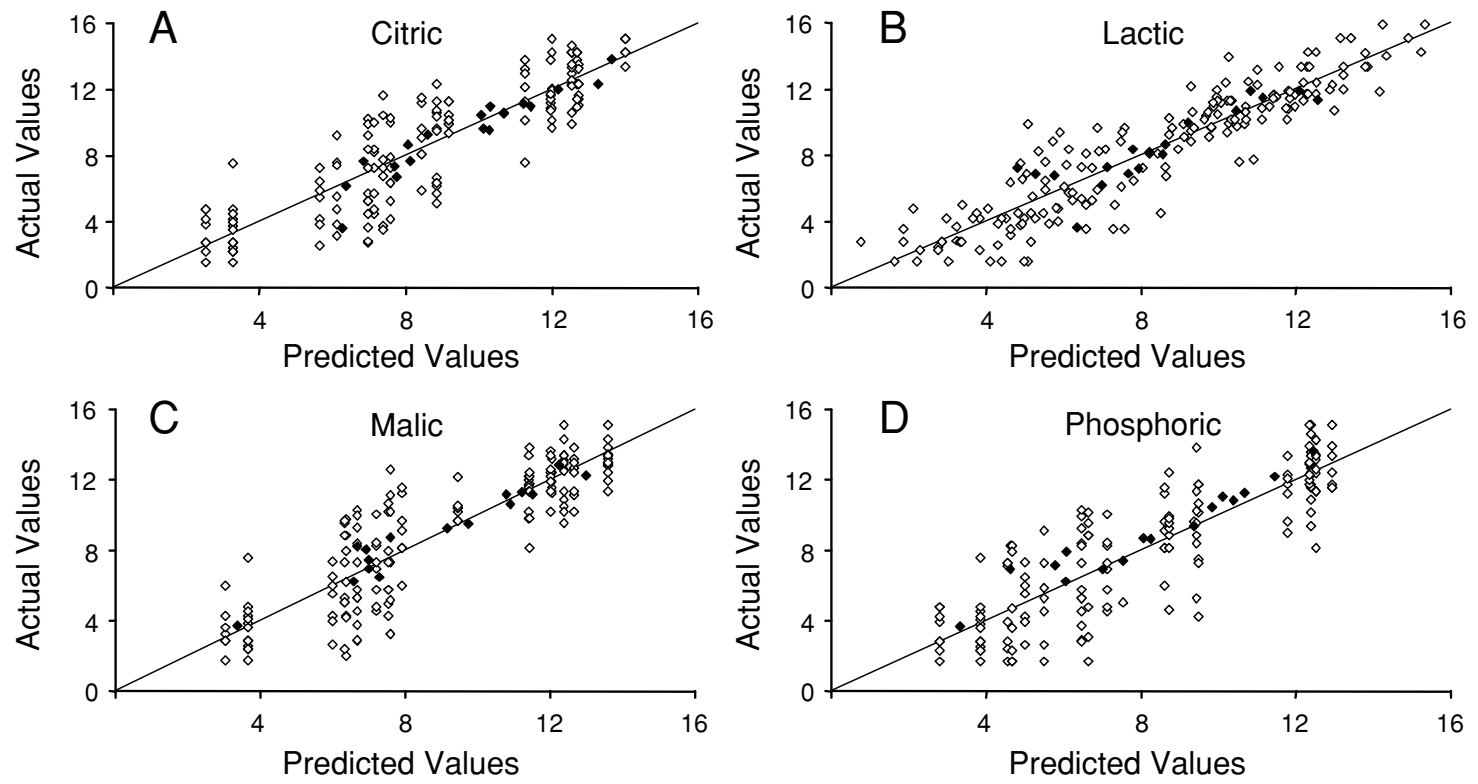

- Model point $\cdot$ Validation points

Figure 7. Perceived strawberry flavor intensity for Group 1: A comparison of predicted values from the models ( $x$-axis) and experimental results ( $y$-axis). Both points used to build the models (open diamonds) and points used as the validation set (filled diamonds) are represented.

one, Lyall, Heck, \& Feldman, 2001) may be other potential explanations. When lactic acid was used, the enhancement contribution of acid was smaller than the sucrose enhancement, suggesting that this acid had less impact on strawberry flavor intensity than did citric and malic acids. Products containing lactic acid are generally perceived to be sweeter than foods containing other acids, because lactic acid provides a milder, more subtle taste sensation. Cayeux and Mercier (2003) reported that at equimolarity, lactic acid was perceived as $20 \%$ less sour and astringent than the three other acids. The results obtained for the four acids seem therefore to be highly correlated with the levels of sourness and/or astringency induced in the solutions, which may originate from acid characteristics as well as from mutual masking between sucrose and acid. This correlation underlines the importance of acid/sucrose ratio to generate optimum perceived strawberry flavor.

\section{GENERAL DISCUSSION}

The effects of sucrose and acid on strawberry flavor intensity were strongly demonstrated in this study. Although not all of the volatiles present in the strawberry aroma were monitored, the release of the volatiles most characteristic of strawberry flavor was shown to be unaffected by the presence of sucrose and/or acid. The observed results are thus unlikely to arise from physicochemical interactions, and could therefore be due to one of several mechanisms.

First, the methodology used may be a source of bias. Because of the lack of an opportunity to rate sourness and sweetness, the panelists may have unintentionally integrated this taste information with strawberry flavor intensity (Frank, van der Klaauw, \& Schifferstein, 1993). However, Stevenson, Prescott, and Boakes (1999) showed that

Table 5

Predicted Perceived Strawberry Flavor Intensity as a Function of Sample Composition and Acid Type for Each Panelist of Group 2

\begin{tabular}{|c|c|c|c|c|c|c|c|c|c|c|}
\hline \multirow[b]{3}{*}{ Acid } & \multicolumn{7}{|c|}{ Square Root (Perceived Strawberry Intensity) $=$} & \multirow[b]{3}{*}{ Adjusted $R^{2}$} & \multirow[b]{3}{*}{ Predicted $R^{2}$} & \multirow{3}{*}{$\begin{array}{l}\text { Adequat } \\
\text { Precisior }\end{array}$} \\
\hline & \multicolumn{3}{|c|}{ Panelist } & \multirow[b]{2}{*}{ * Acid } & \multirow[b]{2}{*}{ * Aroma } & \multirow[b]{2}{*}{ * Acid ${ }^{2}$} & \multirow[b]{2}{*}{ * Aroma $^{2}$} & & & \\
\hline & 1 & 2 & 3 & & & & & & & \\
\hline Citric & 2.85 & 3.22 & 4.62 & 20.4 & 18.8 & -45.9 & & .70 & .66 & 16.3 \\
\hline Malic & 3.30 & 3.53 & 5.33 & 26.4 & 16.3 & -60.1 & & .74 & .70 & 19.5 \\
\hline Lactic & 1.22 & 2.18 & 3.61 & 20.8 & 49.1 & -50.9 & -91.3 & .62 & .56 & 13.4 \\
\hline
\end{tabular}

Note-Each row represents a polynomial equation, with values representing the contribution of each factor. For numerical estimates, the constant value associated with the panelist selected and the specific units for each factor should be employed ( $\% \mathrm{w} / \mathrm{v}$ for acid and $\% \mathrm{v} / \mathrm{v}$ for aroma stimulus). 
Table 6

Predicted Perceived Strawberry Flavor Intensity as a Function of Sample Composition With Phosphoric Acid for Group 2

\begin{tabular}{|c|c|c|c|c|c|c|c|c|}
\hline \multirow[b]{2}{*}{ Panelist } & \multicolumn{5}{|c|}{ Square Root $($ Perceived Strawberry Intensity +2.75$)=$} & \multirow[b]{2}{*}{ Adjusted $R^{2}$} & \multirow[b]{2}{*}{ Predicted $R^{2}$} & \multirow{2}{*}{$\begin{array}{l}\text { Adequate } \\
\text { Precision }\end{array}$} \\
\hline & Constant & * Sucrose & * Acid & * Aroma & $*$ Acid $^{2}$ & & & \\
\hline 1 & 3.25 & 0.129 & 23.8 & 15.5 & -80.3 & .72 & .67 & 17.0 \\
\hline 2 & 3.11 & 0.129 & 28.8 & 15.5 & -80.3 & .72 & .67 & 17.0 \\
\hline 3 & 4.44 & 0.129 & 42.4 & 15.5 & -80.3 & .72 & .67 & 17.0 \\
\hline
\end{tabular}

Note-Each row represents a polynomial equation, with values representing the contribution of each factor. For numerical estimates, the constant value associated with the panelist selected and specific units for each factor should be employed ( $\% \mathrm{w} / \mathrm{v}$ for acid and $\% \mathrm{v} / \mathrm{v}$ for aroma stimulus).

this measurement artifact does not explain all flavor-taste interactions. In addition, Cayeux and Mercier (2003) still observed enhancement effects of acids on the perception of apple, although their participants rated sourness, astringency, and apple flavor intensity. Furthermore, in the present study, a "dumping effect" would not explain the results obtained with Group 2, in which sucrose had no enhancement impact, in contrast with the presence of acid.

The second explanation for apparent interactions between a tastant and an aroma postulates that, although they are anatomically independent systems, taste and aroma can be psychologically integrated (Abdi, 2002). Taste-aroma confusion is one of the manifestations of these perceptual interactions, and some have suggested that it gives rise to the enhancement of taste ratings by aroma stimulus (Frank \& Byram, 1988), because of panelists' inability to attend to the dimension of interest while remaining uninfluenced by other sensory attributes present in the mixture. However true this argument may be for inexperienced panelists, the ability to separate sensations aroused by the various stimuli is commonly recognized among trained panelists. Nevertheless, to what extent this separation can be made is still a controversial subject in research on taste-aroma interactions. In a recent study, Hort and Hollowood (2004) encountered this dilemma when trying to compare variation between experienced and inexperienced panelists in perceived interactions between sucrose and banana aroma stimulus. Whereas for the majority of the trained panel some form of interaction was observed, for a small group, there was no modulation effect of sucrose on the perceived banana intensity. The authors could not relate these findings to variations in training length, nor could they conclude that extensive training would potentially remove the observed effects. The results from the present study therefore cannot be fully explained by simple confusions between the two modalities, and are more likely to arise from other processes.

A further contributing factor for taste-referred aroma enhancement would be the congruency between olfactive and gustatory stimuli, which is defined as the extent to which two stimuli are appropriate for combination in a food product. Indeed, Frank and Byram (1988) demonstrated that taste-induced enhancement of aroma is dependent on the consonance of the aroma and taste stimuli, and this is also true for the interaction of fruitiness and sweetness or sourness. Strawberries naturally contain sugar and acids (citric and malic being, respectively, the predominant and secondary acids). However, phosphoric acid is only found in artificial strawberry-flavored products such as carbonated beverages, and the level of congruency between strawberry flavor and this acid may be questioned. Such a lack of congruency could explain the obtained variations when using this particular acid.

Recent studies have put an emphasis on the central neural integration of the two modalities (taste and aroma) to explain the enhancement/suppression effects of taste on aroma, and vice versa. Commonly known as multisensory convergence, this interpretation relies on the fact that humans are naturally integrative in their sensory perceptions; that is, judgment in one sensory modality is frequently affected by information in other sensory dimensions via coencoding, even if they do not physically or physiologically interact. This interpretation is also supported by neuroimaging investigations. In particular, de Araujo, Rolls, Kringelbach, McGlone, and Phillips (2003) obtained further evidence in favor of the multimodal approach by highlighting brain areas that were activated by both taste and aroma stimuli. Cross-modal integration could therefore be a strong hypothesis to explain the enhancement effects of perceived aroma intensity in these experiments due to the presence of tastants. However, there is no current evidence on the variability of this phenomenon between people.

Learned associations - the process by which an aroma stimulus has become repetitively associated with a particular taste stimulus when experienced retronasally at the same time as the taste-also reportedly give rise to perceptual interactions (Stevenson, Boakes, \& Prescott, 1998; Stevenson, Boakes, \& Wilson, 2000; Stevenson, Prescott, \& Boakes, 1995). Learned associations could account for the observed division of panelists into two groups, one sensitive to both the sweetness of sucrose and the sourness of lactic, malic, or citric acid, and the other not responsive to sucrose variations. This hypothesis states that perception is based on the context in which the flavor was "learned" (Distel \& Hudson, 2001). In this case, some panelists may associate strawberry flavor with fresh fruit from the garden, which tends to be acidic, but others may associate it with jam or other sweet products. The occurrence of enhancement in this complex mixture might therefore depend on cultural and environmental differences, knowledge and experience of the stimuli, and memory and expectations. In addition, physiological and 
behavioral characteristics should not be disregarded as factors inducing variations among panelists. Sensitivity for detecting flavor compounds undeniably varies between individuals and is affected by age, health, and genetics.

There is not one single, simplistic explanation for the results obtained in our experiments. Instead, a convergence of hypotheses indicates that more analysis is needed, especially to understand the main difference between the two behaviors. Globally, these findings suggest that when taste and aroma stimuli are presented together in solution, something more than the sum of the perceptual parts results. The manipulation of acid and sucrose levels, over the ranges tested in these experiments, was shown to have a marked influence on the panelists' perceptions of strawberry flavor. Response surface methodology was found to be a very practical tool to first visualize these effects, then identify the particular rules of combination that lead to flavor magnitude, and thus to determine the optimum formulation of a beverage with regard to the levels of acid and sucrose needed. Since all of the panelists involved in these experiments have been equally trained over the past 4 years, a general pattern of behavior was expected. The observed grouping effects are most likely due to physiological variations, such as stimulus thresholds, or different learned associations between the stimuli. Another question that arises from this study is whether consumers, as opposed to trained panelists, would be able to notice variations in flavor intensity. In addition, it would be interesting to check the validity of our models in a dynamic system, one in which compound concentrations vary over time, and thus an environment closer to the conditions in which foods are eaten, in contrast with the short consumption time of beverages.

\section{REFERENCES}

AвDI, H. (2002). What can cognitive psychology and sensory evaluation learn from each other? Food Quality \& Preference, 13, 445-451.

Bonnans, S., \& Noble, A. C. (1993). Effect of sweetener type and of sweetener and acid levels on temporal perception of sweetness, sourness, and fruitiness. Chemical Senses, 18, 273-283.

Buettner, A., Beer, A., Hannig, C., \& Settles, M. (2001). Observation of the swallowing process by application of videofluoroscopy and real-time magnetic resonance imaging - Consequences for retronasal aroma stimulation. Chemical Senses, 26, 1211-1219.

BuetTNER, A., \& SCHIEBERLe, P. (2000a). Exhaled odorant measurement (EXOM): A new approach to quantify the degree of in-mouth release of food aroma compounds. Lebensmittel Wissenschaft \& Technologie/ Food Science \& Technology, 33, 553-559.

Buettner, A., \& Schieberle, P. (2000b). Influence of mastication on the concentrations of aroma volatiles: Some aspects of flavour release and flavour perception. Food Chemistry, 71, 347-354.

Burdach, K. J., Kroeze, J. H. A., \& Köster, E. P. (1984). Nasal, retronasal, and gustatory perception: An experimental comparison. Perception \& Psychophysics, 36, 205-208.

Calvert, G. A., Spence, C., \& Stein, B. E. (2004). The handbook of multisensory processes. Cambridge, MA: MIT Press.

Cayeux, I., \& Mercier, C. (2003). Sensory evaluation of interaction between smell and taste-Application to sourness. In J. L. Le Quéré \& P. X. Étiévant (Eds.), Flavour research at the dawn of the twenty-first century (pp. 287-292). Paris: Intercept.

Cook, D. J., Davidson, J. M., Linforth, R. S. T., \& Taylor, A. J. (2004). Measuring the sensory impact of flavour mixtures using controlled delivery. In K. D. Deibler \& J. Delwiche (Eds.), Handbook of flavor characterization: Sensory analysis, chemistry, and physiology (pp. 135-150). New York: Dekker.

Cook, D. J., Hollowood, T. A., Linforth, R. S. T., \& TaYlor, A. J. (2002). Perception of taste intensity in solutions of random-coil polysaccharides above and below c*. Food Quality \& Preference, 13, 473480

Cook, D. J., Hollowood, T. A., Linforth, R. S. T., \& Taylor, A. J. (2003). Oral shear stress predicts flavour perception in viscous solutions. Chemical Senses, 28, 11-23.

Cook, D. J., Hollowood, T. A., Pettelot, E., \& Taylor, A. J. (2003). Effects of viscosity on flavor perception: A multimodal approach. In T. Hofmann, C. T. Ho, \& W. Pickenhagen (Eds.), Challenges in taste chemistry and biology (pp. 240-253). Washington, DC: American Chemical Society.

CoOK, D. J., Linforth, R. S. T., \& TAYlor, A. J. (2003). Effects of hydrocolloid thickeners on the perception of savory flavors. Journal of Agricultural \& Food Chemistry, 51, 3067-3072.

Dalton, P., Doolittle, N., Nagata, H., \& Breslin, P. A. S. (2000). The merging of the senses: Integration of subthreshold taste and smell. Nature Neuroscience, 3, 431-432.

Davidson, J. M., Linforth, R. S. T., Hollowood, T. A., \& TAYlor, A. J. (1999). Effect of sucrose on the perceived flavor intensity of chewing gum. Journal of Agricultural \& Food Chemistry, 47, 4336-4340.

de Araujo, I. E. T., Rolls, E. T., Kringelbach, M. L., McGlone, F., \& Phillips, N. (2003). Taste-olfactory convergence, and the representation of the pleasantness of flavour, in the human brain. European Journal of Neuroscience, 18, 2059-2068.

DeSimone, J. A., Lyall, V., Heck, G. L., \& Feldman, G. M. (2001). Acid detection by taste receptor cells. Respiration Physiology, 129, 231-245.

Distel, H., \& Hudson, R. (2001). Judgement of odor intensity is influenced by subjects' knowledge of the odor source. Chemical Senses, 26, 247-251.

EDWARDS, A. E. (1968). Experimental design in psychological research. New York: Holt, Rinehart, \& Winston.

Frank, R. A., \& Byram, J. (1988). Taste smell interactions are tastant and odorant dependent. Chemical Senses, 13, 445-455.

Frank, R. A., van der KlaAuw, N. J., \& Schifferstein, H. N. J. (1993). Both perceptual and conceptual factors influence taste-odor and tastetaste interactions. Perception \& Psychophysics, 54, 343-354.

Friel, E. N., Linforth, R. S. T., \& TAYlor, A. J. (2000). An empirical model to predict the headspace concentration of volatile compounds above solutions containing sucrose. Food Chemistry, 71, 309-317.

Hodgson, M., Linforth, R. S. T., \& TAYlor, A. J. (2003). Simultaneous real-time measurements of mastication, swallowing, nasal airflow, and aroma release. Journal of Agricultural \& Food Chemistry, 51, 5052-5057.

Hollowood, T. A., Davidson, J. M., DeGroot, L., Linforth, R. S. T., \& TAYLOR, A. J. (2002). Taste release and its effect on overall flavor perception. In P. Given \& D. Paredes (Eds.), Chemistry of taste (pp. 166-179). Washington DC: American Chemical Society.

Hollowood, T. A., LinforTh, R. S. T., \& TAYLOR, A. J. (2002). The effect of viscosity on the perception of flavour. Chemical Senses, 27, 583-591.

Hort, J., \& Hollowood, T. A. (2004). Controlled continuous flow delivery system for investigating taste-aroma interactions. Journal of Agricultural \& Food Chemistry, 52, 4834-4843.

Kuo, Y. L., Pangborn, R. M., \& Noble, A. C. (1993). Temporal patterns of nasal, oral, and retronasal perception of citral and vanillin and interaction of these odorants with selected tastants. International Journal of Food Science \& Technology, 28, 127-137.

Murphy, C., \& CaIN, W. S. (1980). Taste and olfaction: Independence vs. interaction. Physiology \& Behavior, 24, 601-605.

Rolls, E. T., \& BaYlis, L. L. (1994). Gustatory, olfactory, and visual convergence within the primate orbitofrontal cortex. Journal of Neuroscience, 14, 5437-5452.

Small, D. M., Voss, J., Mak, Y. E., Simmons, K. B., Parrish, T., \& Gitelman, D. (2004). Experience-dependent neural integration of taste and smell in the human brain. Journal of Neurophysiology, 92, 1892-1903.

Stevenson, R. J., Boakes, R. A., \& Prescott, J. (1998). Changes in odor sweetness resulting from implicit learning of a simultaneous 
odor-sweetness association: An example of learned synesthesia. Learning \& Motivation, 29, 113-132.

Stevenson, R. J., Boakes, R. A., \& Wilson, J. P. (2000). Counterconditioning following human odor-taste and color-taste learning. Learning \& Motivation, 31, 114-127.

Stevenson, R. J., Prescott, J., \& Boakes, R. A. (1995). The acquisition of taste properties by odors. Learning \& Motivation, 26, 433-455.

Stevenson, R. J., Prescott, J., \& Boakes, R. A. (1999). Confusing tastes and smells: How odours can influence the perception of sweet and sour tastes. Chemical Senses, 24, 627-635.

TAYLOR, A. J., \& LiNFORTH, R. S. T. (2003). Direct mass spectrometry of complex volatile and non-volatile flavour mixtures. International Journal of Mass Spectrometry, 223, 179-191.

Taylor, A. J., Linforth, R. S. T., Harvey, B. A., \& Blake, B. (2000). Atmospheric pressure chemical ionisation mass spectrometry for in vivo analysis of volatile flavour release. Food Chemistry, 71, 327-338.

Valdés, R. M., Simone, M. J., \& Hinreiner, E. H. (1956). Effect of sucrose and organic acids on apparent flavor intensity: II. Fruit nectars. Food Technology, 10, 387-390.

(Manuscript received October 27, 2004; revision accepted for publication April 26, 2005.) 